\title{
STRESS FRACTURES OF THE TIBIA IN OSTEOARTHRITIS OF THE KNEE
}

\author{
K. SATKU, V. P. KUMAR, R. W. H. PHO \\ From the National University of Singapore
}

\begin{abstract}
Three women with osteoarthritis of the knee presented after sudden worsening of their symptoms. In each case this was found to be due to a stress fracture of the tibia. With treatment by rest and reduced activity, the fracture healed uneventfully in all three patients.
\end{abstract}

Stress fractures of the tibia are encountered most often in young individuals pursuing athletic or military activities (Devas 1975; Giladi et al. 1985). In elderly people they are much less common, but they have been reported in association with osteoporosis (Devas 1975), rheumatoid arthritis (Wheeldon 1961; Reynolds 1972; Young, Kinsella and Boland 1981) and pyrophosphate arthropathy of the knee (Ross et al. 1983). We describe three cases of stress fractures of the tibia in women with osteoarthritis of the knee.

\section{PATIENTS AND METHODS}

All our patients were women with osteoarthritis of the knee whose pain was controlled by conservative treatment until they developed stress fractures. The serum calcium and phosphate levels were normal in all three. The bone mineral content was assessed by dual photon absorptiometry in Cases 2 and 3 and in both the values were within normal limits for their respective ages.

Case 1. A 72-year-old woman with pain in both knees for two to three years had been treated with analgesics and intra-articular injections. Four weeks before we saw her the pain in her left knee had become severe and, despite medication, had increased until she needed a wheelchair.

The left knee had $5^{\circ}$ of fixed flexion and a $20^{\circ}$ varus deformity, while the right had $5^{\circ}$ of fixed flexion and a $15^{\circ}$ varus deformity. The knees were not warm and there was no effusion. The left knee was tender along the medial and lateral joint lines and over the femoral and

K. Satku, FRCS, Senior Lecturer and Consultant

V. P. Kumar, FRCS, Senior Lecturer and Consultant

P. W. H. Pho, FRCS, Professor

Department of Orthopaedic Surgery, National University of Singapore. Singapore General Hosptial, Singapore 0316, Republic of Singapore.

Requests for reprints should be sent to Mr V. P. Kumar. (C) 1987 British Editorial Society of Bone and Joint Surgery $0301-620 X / 87 / 2063 \$ 2.00$ tibial condyles. There was no gross laxity to suggest a neuropathic joint. The range of movement of the left knee was $5^{\circ}$ to $120^{\circ}$, that of the right was $5^{\circ}$ to $130^{\circ}$. The VDRL was negative, the ESR $80 \mathrm{~mm} / \mathrm{hr}$, and there was nothing to suggest septic arthritis. Radiographs revealed gross degenerative changes and, in addition, a probable stress fracture of the tibia; this was at the lower end of the film and was overlooked at the time (Figs 1 and 2). Two months later when weight-bearing radiographs were taken, the stress fracture was quite obvious (Fig. 3). She was advised to rest and to continue with analgesics. Over the next three months the pain settled and she was again able to walk. One year later she had only occasional pain and needed no medication; a radiograph showed that the stress fracture had healed (Fig. 4).

Case 2. A 54-year-old woman with a history of pain in the left knee for two years had been given indomethacin and intra-articular injections for relief of pain. Two months before consulting us the pain had spontaneously become worse. Clinical examination revealed $5^{\circ}$ of fixed flexion and $5^{\circ}$ of varus deformity but no swelling; the medial femoral and tibial condyles were noted to be tender. The range of movement was $5^{\circ}$ to $130^{\circ}$. Radiographs suggested a possible stress fracture of the tibia (Fig. 5) which was more obvious a month later (Fig. 6).

Three months later her pain had diminshed and she was able to resume normal activities, although she still needed occasional analgesics; the radiological changes are shown in Figures 7 and 8.

Case 3. A 60-year-old woman with bilateral knee pain of one year's duration had $10^{\circ}$ of varus deformity in both knees but no swelling. There was tenderness along the medial joint line. The range of movement was from $0^{\circ}$ to $130^{\circ}$ in both knees, and radiographs of the left knee showed degenerative arthritis. The pain was well controlled with physiotherapy and diclofenac sodium. A repeat radiograph one year later (Fig. 9) was unchanged, but three months after that she suddenly developed much more pain, though the clinical findings were not 


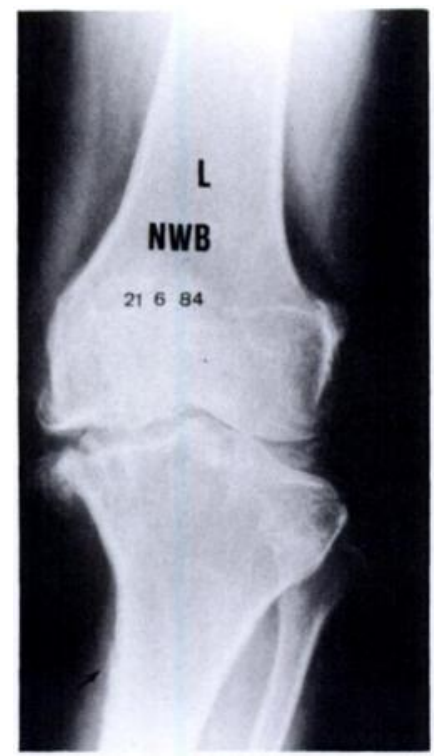

Fig. 1

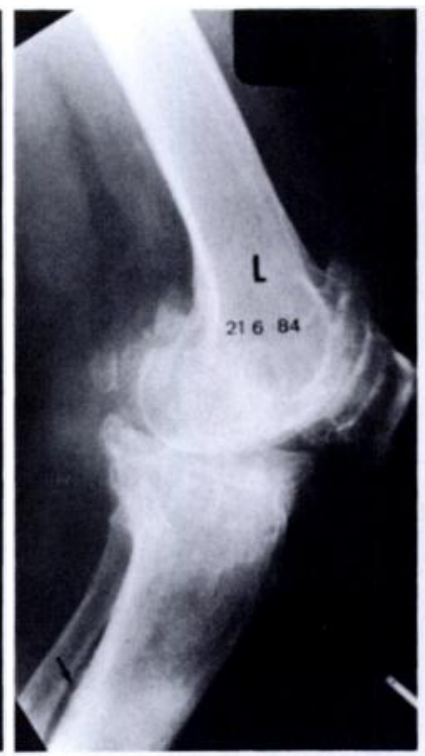

Fig. 2

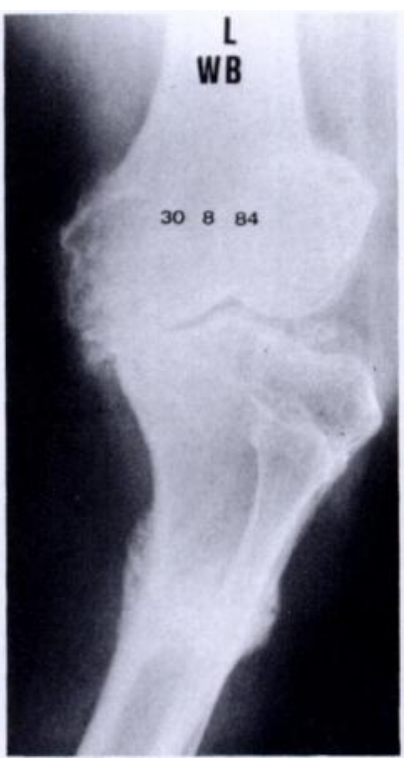

Fig. 3

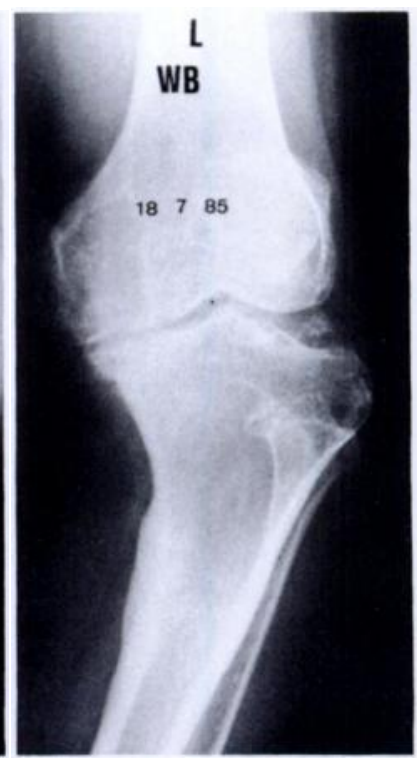

Fig. 4

Case 1 . Figures 1 and 2 - Anteroposterior and lateral radiographs of the left knee; the stress fracture (arrow) is hardly visible and was missed. Figure 3 - Two months later, the radiograph shows an obvious stress fracture. Figure 4 - One year later it is well healed.

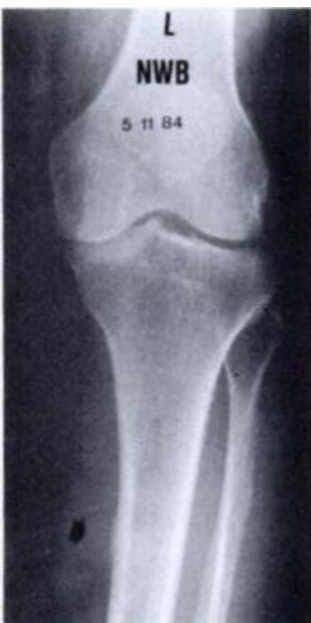

Fig. 5

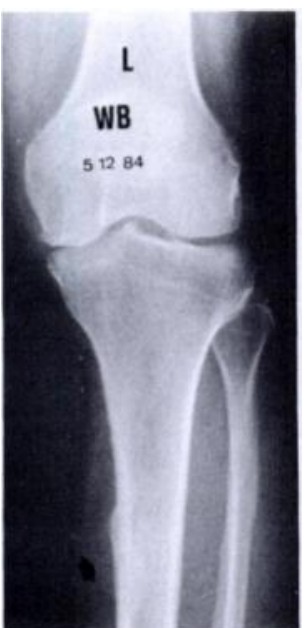

Fig. 6

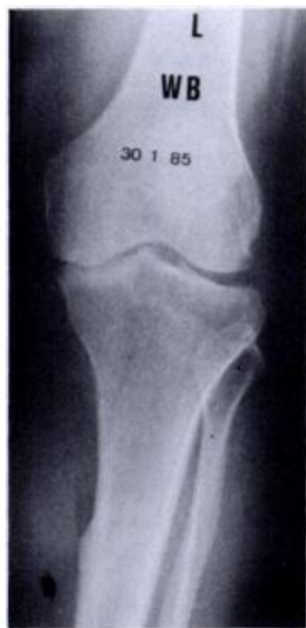

Fig. 7

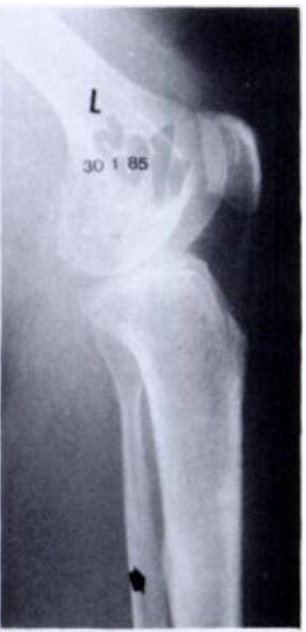

Fig. 8

Case 2. Figure 5 - Anteroposterior radiograph showing a barely visible stress fracture of left tibia (arrow). Figure 6 - One month later the fracture is more obvious. Figures 7 and 8 - Anteroposterior and lateral radiographs three months after the initial radiographs show that there has been no change in the alignment of the tibia at the fracture site.

significantly different. Shortly afterwards, a bone scan revealed increased uptake in the proximal shaft of the tibia. She was advised to reduce activity and to use a frame for walking. Radiographs three months after the onset of her severe pain revealed the stress fracture (Fig. 10). After a further three months her pain diminished, and she has now resumed normal activities.

\section{DISCUSSION}

Despite obvious clinical and radiological evidence of osteoarthritis of the knee, the intensity of pain and functional disability experienced by the patient may vary considerably. Occasionally a patient who has been asymptomatic or whose disability is adequately managed by medical treatment experiences a sudden deterioration; usually this is attributed to deterioration of the arthropathy, or to the development of another lesion such as a tear of a degenerate meniscus, a loose body or osteonecrosis of the femoral or tibial condyles.

Reynolds (1972) reported a single case of stress fracture of the tibia in an elderly patient in association with a varus deformity of the knee, although the cause of the deformity was not discussed. We have been unable to find any reports in the English literature of stress fractures in the proximal shaft of the tibia in association with osteoarthritis of the knee. 


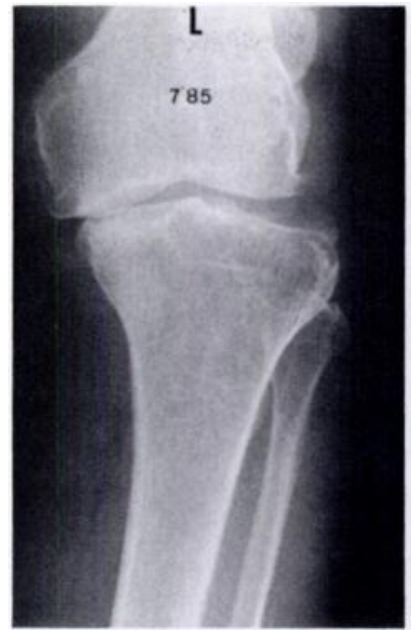

Fig. 9

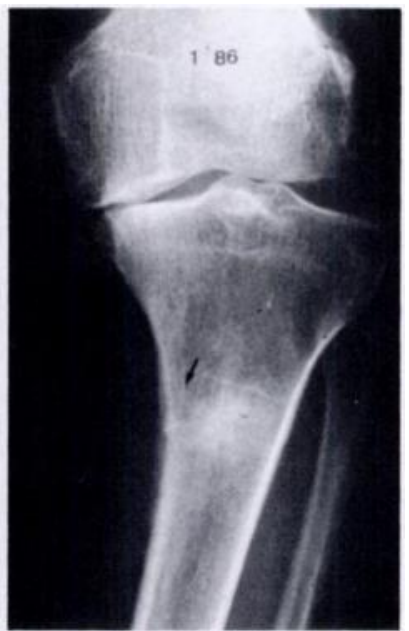

Fig. 10

Case 3. Figure 9 - Anteroposterior radiograph of the knee and tibia three months before a worsening of symptoms. Figure $10-A$ radiograph three months after the symptoms had become more severe shows the stress fracture (arrow).

The patients reported in this paper were seen over a period of two years, and our experience has led us to conclude that when a patient with osteoarthritis of the knee presents with sudden deterioration, the possibility of a stress fracture of the tibia should be considered. All three patients complained of increased pain over the anterior aspect of the knee and tibia, and on initial examination this was attributed to a deterioration of the osteoarthritis.

In Cases 2 and 3 where initial radiographs of the tibia were available, no change in the configuration of the tibia at the fracture site was noted in subsequent films. In Case 1, however, radiographs did not include views of the full extent of the tibia. We emphasise the need for a careful examination of the knee and tibia and full-length radiographs in possible cases of stress fractures. Moreover, since radiological evidence of compression stress fractures may not be obvious until two to three months after the onset of symptoms (Devas 1975), a bone scan may be needed for diagnosis. These compression stress fractures are stable and have little tendency to displace (Devas 1975); if diagnosed early and treated with rest or reduced activity, they heal uneventfully.

We are most grateful to Professor P. Balasubramaniam of the Department of Orthopaedic Surgery, National University of Singapore, for his valuable advice during the writing of the manuscript.

\section{REFERENCES}

Devas M. Stress fractures. Edinburgh etc: Churchill Livingstone, 1975.

Giladi M, Ahronson Z, Stein M, Danon YL, Milgram C. Unusual distribution and onset of stress fractures in soldiers. Clin Orthop 1985; $192: 142-145$.

Ross DJ, Dieppe PA, Watt I, Newman JH. Tibial stress fracture in pyrophosphate arthropathy. J Bone Joint Surg [Br] 1983;65B:474-4.

Reynolds MT. Stress fractures of the tibia in the elderly associated with knee deformity. [Abridged]. J R Soc Med 1972;65:377-80.

Wheeldon FT. Spontaneous fractures in the shin in the presence of knee deformities. J R Soc Med 1961;54:1108.

Young A, Kinsella P, Boland P. Stress fractures of the lower limb in patients with rheumatoid arthritis. J Bone Joint Surg $[\mathrm{Br}] 1981$; 63-B:239-43. 